\title{
Visible Light Adaptive Optics Imaging of the Orion 218-354 Silhouette Disk
}

\author{
Katherine B. Follette ${ }^{1,5}$, Laird M. Close ${ }^{1}$, Jared R. Males ${ }^{1,4}$, Derek \\ Kopon $^{2}$, Ya-Lin $\mathbf{W u}^{1}$, Katie M. Morzinski ${ }^{1,4,5}$, Philip Hinz. ${ }^{1}$, Timothy \\ J. Rodigas ${ }^{1}$, Alfio Puglisi ${ }^{3}$, Simone Esposito ${ }^{3}$, Armando Riccardi ${ }^{3}$, \\ Enrico Pinna ${ }^{3}$, Marco Xompero ${ }^{3}$, \& Runa Briguglio ${ }^{3}$ \\ ${ }^{1}$ Steward Observatory, The University of Arizona, 933 N Cherry Ave, Tucson, AZ 85721, USA \\ ${ }^{2}$ Max Planck Institute for Astronomy, Königstuhl 17, 69117 Heidelberg, Germany \\ ${ }^{3}$ INAF - Osservatorio Astrofisico di Arcetri, Largo E. Fermi 5, I-50125, Firenze, Italy \\ ${ }^{4}$ NASA Sagan Fellow \\ ${ }^{5}$ kfollette@as.arizona.edu
}

\begin{abstract}
We present the first ground-based adaptive optics images of a silhouette disk. This disk, Orion 218-354, is seen in silhouette against the bright nebular background of Orion, and was resolved using the new Magellan Adaptive Secondary AO system and its VisAO camera in Simultaneous Differential Imaging (SDI) mode. PSF subtraction of Orion 218-354 reveals a disk $\sim 1 "$ (400 AU) in radius, with the degree of absorption increasing steadily towards the center of the disk. By virtue of the central star being unsaturated, these data probe inward to a much smaller radius than previous HST images. Our data present a different picture than previous observers had hypothesized, namely that the disk is likely optically thin at $\mathrm{H} \alpha$ at least as far inward as $20 \mathrm{AU}$. In addition to being among the first high-resolution $\mathrm{AO}$ images taken in the optical on a large telescope, these data reveal the power of SDI imaging to illuminate disk structure, and speak to a bright future for visible AO imaging. Analysis of the results described briefly here can be found in full detail in Follette et al. (2013).
\end{abstract}

Keywords. Instrumentation: Adaptive Optics, Planetary Systems: Protoplanetary Disks

\section{Introduction}

Silhouette disks have long been of interest to the astronomical community as they provide perhaps the most unequivocal direct evidence of the existence of dusty circumstellar disks around young stars, as well as some of the most robust measurements of disk extents and inclinations. To date, all observations of these disks in silhouette have been done from space with HST. Similar observations have been precluded from the ground by a number of factors, chief among them the inability of most AO systems to achieve them. In particular, the wavelengths of the nebular lines necessary to see these disks in silhouette all lie shortward of $1 \mu \mathrm{m}$, well outside the operating wavelengths of most AO systems. The central stars in these disks are also fainter than is manageable with traditional wavefront sensors. MagAO is the first AO system capable of imaging these disks from the ground. It is able to do so by virtue of its adaptive secondary mirror, which allows for correction on the necessary spatial scales to achieve AO imaging in the visible, as well as its pyramid wavefront sensor, which allows for binning of the pyramid pupils to lock on fainter natural guide stars. 

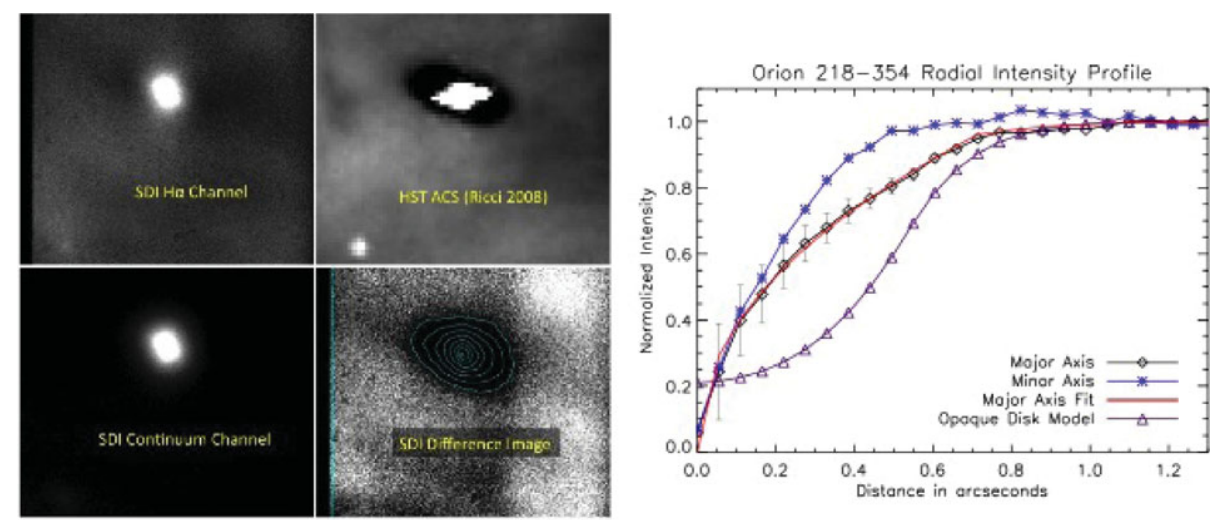

Figure 1. Left panel: Upper left: MagAO $\mathrm{H} \alpha$ image, showing the central star and the outer regions of the disk seen in silhouette. Lower left: MagAO SDI continuum (643 nm) image. Lower right: SDI difference image ( $\mathrm{H} \alpha$ minus scaled continuum), revealing the entire disk in silhouette. Contours denote levels of decreasing flux moving towards the center of the disk. Upper right: HST ACS image of the same disk on the same physical scale from Ricci (2003).Right panel: Radial profile of MagAO ADI difference image, as well as model fits and the convolved best-fit optically thick disk model of McCaughrean \& O'dell (1996), which is clearly a poor match to the data

\section{Observations}

We observed the silhouette disk Orion 218-354 with MagAO's visible light science camera, VisAO, on December 6, 2012 as part of the commissioning effort of the system (Close et al. (2013)). The disk was observed in simultaneous differential imaging (SDI) mode, in which a Wollaston prism splits the beam into two beams of equal brightness (for unpolarized sources). Each of these is passed through a different narrowband filter. One filter is centered on a spectral line of interest $(\mathrm{H} \alpha,[\mathrm{OI}]$ or $[\mathrm{SII}])$, and one on the neighboring continuum. This allows for simple, robust PSF subtraction through the nearly identical simultaneous continuum channel PSF.

\section{Conclusions}

From the Orion 218-354 data, we conclude that the radial intensity profile of this disk is suggestive of a small optical depth at $\mathrm{H} \alpha$, a surprising result given previous HST work suggesting that all of the silhouette disks are optically thick (MacCaughrean (1996)). We convolve the best fit optically thick disk model of earlier observers with our continuum PSF and find that it is inconsistent with our data both in an absolute sense and in its general shape and character. In Follette et al. (2013), we use the observed profile to estimate the disk extent, geometry, small grain dust mass and radial mass density profile, and use these as inputs to the Whitney Monte Carlo radiative transfer code to generate the first multiwavelength model of the Orion 218-354 SED.

\section{References}

Close, L. M., et al., 2013, ApJ, 774, 94

Follette, K. B., et al., 2013, ApJL, 775, 113

McCaughrean, M. J. \& O'dell, C. R. 1996, AJ, 111, 1977

Ricci, L., Robberto, M., \& Soderblom, D. R. 2008, AJ, 136, 2136 